Matthew Foreman $\cdot$ Benjamin Weiss

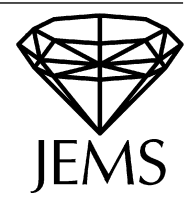

\title{
An anti-classification theorem for ergodic measure preserving transformations
}

Received February 20, 2003 and in revised form September 26, 2003

Abstract. Despite many notable advances the general problem of classifying ergodic measure preserving transformations (MPT) has remained wide open. We show that the action of the whole group of MPT's on ergodic actions by conjugation is turbulent in the sense of G. Hjorth. The type of classifications ruled out by this property include countable algebraic objects such as those that occur in the Halmos-von Neumann theorem classifying ergodic MPT's with pure point spectrum. We treat both the classical case of $\mathbb{Z}$ as well as the case of general countable amenable groups.

\section{Introduction}

Dynamical systems are often studied from a probabilistic or statistical point of view by finding an ergodic invariant probability measure on the space. This allows qualitative descriptions of the dynamical system such as being "truly random" (e.g. Bernoulli) or truly deterministic (e.g. 0-entropy).

The probabilistic behavior of many classical dynamical systems has been characterized precisely by determining the invariant measure up to measure theoretic isomorphism. This led to the general project of classifying measure preserving systems up to isomorphism. Despite dramatic successes, notably Ornstein's theorem classifying Bernoulli transformations in terms of entropy [11], and the Halmos-von Neumann theorem classifying ergodic translations on compact groups, this project largely remains unfulfilled.

Indeed, there has been some accumulation of evidence suggesting that such a classification is impossible. The main result of this note is a precise statement of such a theorem.

As a corollary of the main result, it is impossible to effectively associate to members of a generic collection of ergodic measure preserving transformations countable algebraic structures (e.g. groups) in such a way that two transformations are isomorphic just in case the associated algebraic objects are isomorphic. (In other words, there is no Borel functor from the category of ergodic measure preserving transformations to the category of (say) countable groups.)

M. Foreman: Department of Mathematics, University of California at Irvine, Irvine, CA 92697, USA; e-mail: mforeman@math.uci.edu

B. Weiss: Institute of Mathematics, Hebrew University of Jerusalem, Jerusalem, 91904 Israel; e-mail: weiss@math.huji.ac.il 
There have been some precursors to this result. For example Hjorth ([8]) showed the analogous result for measure distal transformations of height 2 . We will use the notion of turbulence defined by Hjorth for our theorem.

We begin by giving some terminology.

Definition 1. Let $X, Y$ be Polish spaces, and $E, F$ be equivalence relations on $X$ and $Y$ respectively. Then $E$ is Borel reducible to $F\left(E \leq_{B} F\right)$ iff there is a Borel measurable function $f: X \rightarrow Y$ such that for all $x_{1}, x_{2} \in X$ :

$$
x_{1} E x_{2} \text { iff } f\left(x_{1}\right) F f\left(x_{2}\right) .
$$

In other words, any question about $E$ can be "reduced" via $f$ to a question about $F$. There is an obvious generalization of this notion to equivalence relations on Borel subsets $A \subset X, B \subset Y$.

In this language, the classical result that any two perfect Polish spaces are Borel isomorphic can be interpreted as saying that for all perfect Polish $X$ and $Y$, there is a Borel bijection $f: X \rightarrow Y$ such that $f$ reduces the relation of equality on $X$ to the relation of equality on $Y$ and $f^{-1}$ reduces equality on $Y$ to equality on $X$. Thus the question of attaching complete invariants that come from an arbitrary Polish space is not more general than the issue of attaching complete numerical invariants.

If we let $E_{0}$ be the equivalence relation on $2^{\mathbb{N}}$ (infinite sequences of 0 's and 1's) defined by setting $\vec{x} \sim \vec{y}$ in case there is an $N$ such that $x_{n}=y_{n}$ for all $n>N$, then classical 0-1 laws show that there is no Borel reduction of $E_{0}$ to equality on a Polish space. (There are converses to this, see [5].)

As remarked by Feldman ([1]) the following result implies that there can be no numerical invariants attached to $\mathcal{K}$-automorphisms:

Theorem (Ornstein-Shields). There is a Borel reduction of $E_{0}$ to the isomorphism equivalence relation on the $\mathcal{K}$-automorphisms.

In particular, there is no numerical invariant of $\mathcal{K}$-automorphisms analogous to entropy.

On the other hand, the Halmos-von Neumann theorem is considered to give a suitable structure theorem for the discrete spectrum transformations in spite of the fact that the equivalence relation $E_{0}$ is also reducible to isomorphism of discrete spectrum transformations [2].

The Halmos-von Neumann theorem associates to each ergodic discrete spectrum transformation a countable subset of the unit circle in such a way that two transformations are isomorphic iff the associated countable sets are equal. By using predicates for rational intervals, one can effectively associate to each countable subset of the unit circle a countable structure in a manner that reduces equality of countable sets to isomorphism of the countable structures. Until this paper there was no clear result showing that this was not possible for a collection of generic measure preserving transformations (e.g. the weakly mixing transformations).

To establish such a result we use some notions of Hjorth [6]. Let $S_{\infty}$ be the group of permutations of $\mathbb{N}$ with the topology of pointwise convergence. We will consider continuous actions of $S_{\infty}$ on Polish spaces. 
This example is chosen specifically because it includes the isomorphism relations on countable algebraic objects. We illustrate this in a particular case:

Example 2. Let $X$ be the space of functions $f: \mathbb{N} \times \mathbb{N} \times \mathbb{N} \rightarrow 2$ with the obvious product topology. Let $S_{\infty}$ act on $X$ by permuting all three copies of $\mathbb{N}$ simultaneously. If $G$ is a countable group ( say with universe $\mathbb{N}$ and group operation $\odot$ ) we can associate an element $f_{G} \in X$ by setting

$$
f_{G}(l, m, n)=1 \quad \text { iff } \quad l \odot m=n .
$$

If we equip the space of functions $f: \mathbb{N} \times \mathbb{N} \times \mathbb{N} \rightarrow 2$ with the "usual" topology of pointwise convergence (i.e. the $\mathbb{N} \times \mathbb{N} \times \mathbb{N}$ product topology of the discrete space $2=\{0,1\})$ then the collection of $f_{G}$ for $G$ a countable group is a dense $\mathcal{G}_{\delta}$ set, and hence the usual topology restricted to the $f_{G}$ 's is a Polish topology.

If $G, H$ are two groups with universe $\mathbb{N}$, then $G \cong H$ iff there is a $g \in S_{\infty}$ such that

$$
g f_{G}=f_{H} .
$$

We leave it to the reader to see that isomorphism on countable rings, rings with an ordering, fields etc. are all $S_{\infty}$-actions. (Indeed, there is a universal such action, the logic action.)

To show that there is no way of computing isomorphism classes of countable structures as a complete invariant, it suffices to show that the associated equivalence relation is not reducible to an $S_{\infty}$-action. A mechanism for doing this was developed by Hjorth [6].

Definition 3. Let $G$ be a Polish group acting continuously on a Polish space $X$. Then the action is turbulent iff:

(1) Every orbit is dense.

(2) Every orbit is meager.

(3) For all $x, y \in X, V \subset X, U \subset G$ open, with $x \in V, 1 \in U$, there exist $y_{0} \in[y]_{G}$ and $\left\langle g_{i}\right\rangle_{i \in \mathbb{N}} \subset U,\left\langle x_{i}\right\rangle_{i \in \mathbb{N}} \subset V$ such that
(a) $x_{0}=x$,
(b) $x_{i+1}=g_{i} x_{i}$,
(c) there is a subsequence $i_{n}$ such that $x_{i_{n}}$ converges to $y_{0}$.

Hjorth proved the following result:

Theorem. Suppose that the action of $G$ on $X$ is turbulent. Then the orbit equivalence relation ( $x E y$ iff there is a $g \in G$ such that $g x=y$ ) cannot be reduced to an $S_{\infty}$-action.

Hjorth's result clearly implies that if the action of $G$ is turbulent, there is no invariant comeager set on which the orbit equivalence relation can be reduced to any $S_{\infty}$-action. Hence the conclusion is "generic".

The notion of turbulence almost exactly captures the combinatorial content of not being an $S_{\infty}$-action: Hjorth showed that for a Borel equivalence relation $E, E$ is not reducible to an $S_{\infty}$-action just in case there is a turbulent action reducible to $E$. Rather embarassingly, we do not know whether isomorphism for ergodic measure preserving transformations is a Borel equivalence relation. 
We will use the following lemma (whose proof is left to the reader), which gives a sufficient condition that is easier to verify:

Lemma 4. Suppose that $G$ is a Polish group acting continuously on a Polish space X. Suppose further that:

(1) Every orbit is dense.

(2) Every orbit is meager.

(3) For all $x \in X$ and all neighborhoods $V_{0} \subset X$ of $x$ and all neighborhoods $U \subset G$ with $1 \in U$ there is a $V \subset V_{0}$ with $x \in V$ such that for all $y \in V$ and all neighborhoods $W$ of $y$, there are finite sequences $\left\langle g_{i}\right\rangle_{i \leq N-1} \subset U,\left\langle x_{i}\right\rangle_{i \leq N-1} \subset V$ with
(a) $x_{0}=x$,
(b) $x_{i+1}=g_{i} x_{i}$,
(c) $x_{N} \in W$.

Then the action is turbulent.

In order to apply this technology we must describe a Polish group acting continuously on a Polish space. (See e.g. [2] for a laborious development of this situation.)

Since every separable non-atomic probability measure space is isomorphic to the unit interval with Lebesgue measure, every ergodic, aperiodic measure preserving transformation on a probability space is isomorphic to an ergodic transformation on $[0,1]$ and gives rise to a unitary operator on $L^{2}([0,1])$. (We identify measure preserving transformations that agree on a set of full measure.) The space of unitary operators arising from measure preserving transformations is a Polish group when endowed with the weak operator topology. Halmos ([4]) showed that the ergodic measure preserving transformations are a dense $\mathcal{G}_{\delta}$ subset of the measure preserving transformations, and hence form a Polish space.

Moreover, the relation of isomorphism between measure preserving transformations is given by the action of conjugacy of the measure preserving transformations on the ergodic transformations.

This context can be generalized to show that if $H$ is a fixed countable amenable group then there is a natural Polish topology on the space of $H$-actions on $[0,1]$ by measure preserving transformations. (We give details in Section 4.) Moreover the action of conjugation by the group of measure preserving transformations on the space of $H$-actions again coincides with isomorphism.

We prove:

Theorem A. Let $H$ be a countable amenable group. Let $X$ be the space of ergodic actions of $H$ on $[0,1]$. Let $G$ be the group of measure preserving transformations acting on $X$ by conjugation. Then this action is turbulent.

An important special case of this is:

Theorem B. Let $G$ be the group of measure preserving transformations of $[0,1]$ acting by conjugation on $X$, the space of ergodic measure preserving transformations. Then the action of $G$ is turbulent.

We note that Hjorth [7] has proved that it is possible to reduce a turbulent equivalence relation to the space of ergodic actions of any countable discrete group that is not "abelian-by- 
finite". Hence it is not possible to classify the measure preserving actions of any countable group by using countable algebraic objects.

We begin by proving Theorem B, and indicate how to strengthen the proof in Section 4 The proof is somewhat different than the proof of the more general result, Theorem $\mathrm{A}$. We present it because it gives slightly more information than the proof of Theorem A.

We need to concretely describe a neighborhood basis for the measure preserving transformations. The following description can be found in [4].

Definition 5. For each measure preserving transformation $T$ and finite sequence of measurable sets $A_{0}, \ldots, A_{n-1}$ and $\epsilon>0$, let $N\left(A_{0}, \ldots, A_{n-1}, \epsilon\right)$ be the collection of all $S$ such that for all $i<n$ the measure of $S\left(A_{i}\right) \triangle T\left(A_{i}\right)$ is less than $\epsilon$.

Proposition 6. Let $T$ be a measure preserving transformation of $[0,1]$. Then the collection $\left\{N\left(A_{0}, \ldots, A_{n-1}, \epsilon\right): A_{i}\right.$ is measurable, $\left\{A_{i}\right\}$ is a partition of $[0,1]$ and $\left.\epsilon>0\right\}$ forms a neighborhood basis for $T$.

In [4], Halmos defines a metric on the space of measure preserving transformations by setting $d^{\prime}(S, T)$ to be the measure of $\{x: S(x) \neq T(x)\}$. The topology of this metric is finer than the Polish topology described above. Some of our results can be restated using the $d^{\prime}$ metric.

The prerequisites from ergodic theory are very mild. Throughout we will be assuming our measure space is $[0,1]$ and we will denote the Lebesgue measure by $\mu$. We heavily use the following fact:

Rokhlin's Lemma I. Let $T$ be an ergodic measure preserving transformation on [0, 1], $N \in \mathbb{N}, \epsilon>0$. Then there is a set $B \subset[0,1]$ such that:

(1) $B, T B, T^{2} B, \ldots, T^{N-1} B$ are pairwise disjoint.

(2) The measure of $\bigcup_{i<N} T^{i} B$ is at least $1-\epsilon$.

As is standard, we will call $B, T B, T^{2} B, \ldots, T^{N-1} B$ the levels of an $(N, \epsilon)$ Rokhlin tower with base $B$. We will call $X \backslash \bigcup_{i<N} T^{i} B$ the remainder of the tower.

This lemma can be refined using the ergodic theorem to yield:

Rokhlin's Lemma II. Let $T$ be an ergodic measure preserving transformation on [0, 1], $\epsilon>0$ and $\left\{A_{1}, \ldots, A_{n}\right\}$ be subsets of $[0,1]$. Then there is an $M$ and for all $N>M$ there is a set $B \subset[0,1]$ such that:

(1) $B, T B, T^{2} B, \ldots, T^{N-1} B$ are pairwise disjoint.

(2) The measure of $\bigcup_{i<N} T^{i} B$ is at least $1-\epsilon$.

(3) For all $y \in B$ and $j<n$,

$$
\left|\#\left\{i<N: T^{i} y \in A_{j}\right\} / N-\mu\left(A_{j}\right)\right|<\epsilon .
$$

Notation. For a point $y \in[0,1]$ and a transformation $T$ we will often write $y^{i}$ for the point $T^{i} y$. We will confuse the reader by using absolute value symbol " $|C|$ " both for the cardinality of $C$ if $C$ is a set, and for the absolute value of $C$ if $C$ is a number. 


\section{A useful lemma}

We start with a definition:

Definition 7. We will say that a measure preserving transformation $T^{\prime}$ has the same orbits almost everywhere as $T$ does if for almost all $x$ the $T^{\prime}$-orbit of $x$ is equal to the $T$-orbit of $x$.

In this section we prove:

Lemma 8. Suppose that $T$ is ergodic, $\epsilon>0$ and $\left\{C_{i}\right\}_{i<k},\left\{D_{i}\right\}_{i<k}$ are two measurable partitions of $[0,1]$ such that for each $i<k, C_{i}$ and $D_{i}$ have the same measure. Then there is a $T^{\prime}$ that has the same orbits almost everywhere as $T$ and is such that for all $i$ the measure of $T^{\prime}\left(C_{i}\right) \triangle D_{i}$ is less than $\epsilon$.

From this lemma and the definition of the basic open neighborhoods, it is easy to see:

Lemma 9. Let $S, T$ be ergodic measure preserving transformations and $U$ a neighborhood of $S$. Then there is a $T^{\prime} \in U$ that has the same orbits almost everywhere as $T$.

Proof of Lemma 8. Fix an $\epsilon>0$ and partitions $\left\{C_{l}\right\}_{l<k},\left\{D_{l}\right\}_{l<k}$, and let $N$ be large enough and $\delta$ small enough that $(2 k+2) \delta+(2 k+4) / N<\epsilon$. Choose an $(N, \delta)$ Rokhlin tower with base $B_{0}$ and remainder $R$ such that for all $y$ in the base and $l<k$, the number of elements of $C_{l} \cap\left\{y^{i}\right\}_{i=1}^{N-2}$ differs from the number of elements of $D_{l} \cap\left\{y^{i}\right\}_{i=1}^{N-2}$ by less than $\delta N$.

We start by fixing an arbitrary $y$ in the base of the tower and building a sequence of partial functions $t_{0} \subset t_{1} \subset \cdots \subset t_{k}$ from $\left\{y^{i}\right\}_{i=1}^{N-2}$ to $\left\{y^{i}\right\}_{i=1}^{N-2}$ such that

- $t_{l}$ is a one-to-one partial function from $\bigcup_{j<l} C_{j}$ to $\bigcup_{j<l} D_{j}$ sending the elements of $C_{j}$ in its domain to elements of $D_{j}$.

- The graph of $t_{l}$ contains no cycles.

- The domain of $t_{k}$ contains more than $N-k(\delta+1) N-2$ points.

This is done inductively by setting $t_{0}=\emptyset$ and obtaining $t_{l}$ from $t_{l-1}$ by taking $t_{l}$ to be a maximal extension of $t_{l-1}$ such that $t_{l}$ maps $C_{l-1}$ to $D_{l-1}$ and has no cycles in its graph.

We note that there are at most $\max \left\{\left|C_{l-1} \cap\left\{y^{i}\right\}_{i=1}^{N-2}\right|-\left|D_{l-1} \cap\left\{y^{i}\right\}_{i=1}^{N-2}\right|+1,1\right\}$ points of $C_{l-1} \cap\left\{y^{i}\right\}_{i=1}^{N-2}$ left out of the domain of $t_{l}$. (If there were at least two points of $D_{i}$ not in the range of $t_{l}$ then it would be possible to extend $t_{l}$ without creating a cycle.) Since the distributions of $C_{l-1}$ and $D_{l-1}$ are equal except for an error of proportion $\delta, t_{i}$ is defined on all of $C_{l-1} \cap\left\{y^{i}\right\}_{i=1}^{N-2}$ except perhaps $N \delta+1$ points.

Let $L_{l}$ be the set of points of $C_{l} \cap\left\{y^{i}\right\}_{i=1}^{N-2}$ not in the domain of $t_{l}$. Then $\bigcup_{l<k} L_{l}$ has cardinality less than $k(N \delta+1)$. Thus we have constructed $t_{k}$ as desired.

Now extend $t_{k}$ to a total function $t_{y}$ from $\left\{y^{i}\right\}_{i=0}^{N-2}$ to $\left\{y^{i}\right\}_{i=1}^{N-1}$ with no cycles in its graph such that $t^{N-1}(y)=y^{N-1}$. Define $s_{y}:\{0, \ldots, N-2\} \rightarrow\{1, \ldots, N-1\}$ by setting $s_{y}(i)=j$ iff $t_{y}\left(y^{i}\right)=y^{j}$.

Since there are only finitely many such $s_{y}$ 's we can partition the base $B_{0}$ of the Rokhlin tower into measurable sets by setting $B_{s}=\left\{y \in B_{0}: s_{y}=s\right\}$.

Define a measure preserving transformation $T^{\prime}$ as follows: 
(1) $T^{\prime} \uparrow\left(R \cup T^{N-1} B_{0}\right)=T\left\lceil\left(R \cup T^{N-1} B_{0}\right)\right.$

(2) For $y \in B_{s}$ and $j<N-1$ we let $T^{\prime}\left(y^{j}\right)=y^{s(j)}$.

In other words, on each $T$-fiber above $y \in B_{0}$ we follow $t_{y}$, and outside the tower and on the top level of the tower we follow $T$. Since $T^{\prime}$ respects the columns of the Rokhlin tower and the entry and exit levels of the tower, it must have the same orbits as $T$.

To see that $T^{\prime}$ suffices: for each $k$, on each $B_{s}$ the measure of the set of points $x \in C_{i}$ in the tower above $B_{s}$ where $T^{\prime} x \notin D_{i}$ is at most $\mu\left(B_{S}\right)(k(N \delta+1)+2)$. Hence the total measure of points $x \in C_{i}$ in the tower where $T^{\prime} x \notin D_{i}$ is at most $k(\delta+1 / N)+2 / N$. Since the remainder $R$ has measure at most $\delta$ the total measure of $T\left(C_{i}\right) \triangle D_{i}$ is at most $2 \delta+2 k(\delta+1 / N)+4 / N=(2 k+2) \delta+(2 k+4) / N$.

It is well known, and easily established by an exhaustion argument, that if sets $A$ and $B$ have the same measure then there is a generalized power of $T$ that maps $A$ to $B$. Doing this for two partitions yields an element in the full group of $T$, but its orbits will, in general, only be finite parts of the $T$-orbits. The proof of Lemma 8 can be extended to prove the following:

Remark 10. Suppose that $T$ is ergodic and $\left\{C_{i}\right\}_{i \in \mathbb{N}},\left\{D_{i}\right\}_{i \in \mathbb{N}}$ are two measurable partitions of $[0,1]$ such that for each $i \in \mathbb{N}, C_{i}$ and $D_{i}$ have the same measure. Then there is a $T^{\prime}$ such that for a.e. $x$ the $T^{\prime}$-orbit of $x$ is infinite and included in the $T$-orbit of $x$ and is such that for all $i, T^{\prime}\left(C_{i}\right)=D_{i}$.

Proof (sketch). The main point is a refinement lemma:

Lemma 11. Let $T$ be an ergodic measure preserving transformation, $\epsilon>0$, and $l \in \mathbb{N}$. Let $\left\{C_{i}: i \in \mathbb{N}\right\}$ and $\left\{D_{i}: i \in \mathbb{N}\right\}$ be two measurable partitions such that the measure of $C_{i}$ is equal to the measure of $D_{i}$. There is a $T^{\prime}$ which has the same orbits almost everywhere as $T$ does and such that:

(1) For all $i$, if $x \in C_{i}$ and $T(x) \in D_{i}$ then $T^{\prime}(x)=T(x)$.

(2) For all $i<$ l the measure of $T\left(C_{i}\right) \triangle D_{i}$ is less than $\epsilon$.

This refinement lemma is proved analogously to Lemma 8 . We choose a Rokhlin tower to respect the distribution of the partitions $\left\{C_{i}: i<l\right\} \cup\left\{[0,1] \backslash \bigcup_{i<l} C_{i}\right\}$ and $\left\{D_{i}: i<l\right\} \cup$ $\left\{[0,1] \backslash \bigcup_{i<l} D_{i}\right\}$. We then let $G=\left\{x:\right.$ for all $i$, if $x \in C_{i}$ then $\left.T(x) \in D_{i}\right\}$ and perform the same construction, except that we start with $t_{0}\left\lceil\left(G \cap\left\{y^{i}\right\}_{i=0}^{N-2}\right)=T \uparrow\left(G \cap\left\{y^{i}\right\}_{i=0}^{N-2}\right)\right.$. (If $y^{0} \notin G$, then each $t_{i}$ has domain contained in $\left\{y^{i}\right\}_{i=1}^{N-2}$, as before.)

To finish the proof of Remark 10, repeatedly use the refinement lemma to build a sequence of transformations $T_{0}, T_{1}, \ldots, T_{j}, \ldots$ with $\epsilon_{j} \rightarrow 0$ and $l_{j} \rightarrow \infty$, making sure that $T_{j+1}\left\lceil G_{j}=T\left\lceil G_{j}\right.\right.$, where $G_{j}=\left\{x\right.$ : for all $i$, if $x \in C_{i}$ then $\left.T_{j}(x) \in D_{i}\right\}$. Let $T^{\prime}$ be the measure preserving transformation such that for all $j$ and $x \in G_{j}, T^{\prime}(x)=T_{j}(x)$.

\section{The main result}

In this section we prove Theorem B. 
Theorem 12. Let $G$ be the group of measure preserving transformations of $[0,1]$ acting by conjugation on $X$, the space of ergodic measure preserving transformations. Then the action of $G$ is turbulent.

Corollary 13. There is no dense $\mathcal{G}_{\delta}$ subset of the measure preserving transformations on which the equivalence relation of isomorphism can be reduced to an $S_{\infty}$-action.

Proof of Theorem 12. In fact we will prove a stronger result about the metric $d^{\prime}$ :

Claim 14. Suppose that $\epsilon, \delta>0, S, T$ are ergodic and $S$ has the same orbits almost everywhere as $T$ does. Then there is a sequence of elements $g_{i} \in G$ for $i<n$ such that:

(1) $\left\{x: g_{i}(x) \neq x\right\}$ has measure less than $\epsilon$,

(2) if $T_{0}=T, T_{i+1}=g_{i} T_{i}$ then for all $i,\left\{x: T_{i}(x) \notin\{T(x), S(x)\}\right\}$ has measure less than $\delta$,

(3) $\left\{x: T_{n}(x) \neq S(x)\right\}$ has measure less than $\delta$.

Using Lemma 4 , and assuming the claim, we now verify that the action is turbulent.

We note first that Rokhlin's lemma implies that every transformation is $d^{\prime}$-close to periodic transformations of sufficiently large period and small remainder. Since any two periodic transformations of the same period are conjugate, we see that every orbit is dense.

The fact that every orbit is meager follows easily from [9], where it is shown that for ergodic transformations $T$, the collection of ergodic transformations disjoint from $T$ is a dense $\mathcal{G}_{\delta}$. If there were a non-meager orbit, it would have to be comeager, since it is invariant. But then a transformation $T$ in the orbit would have to be disjoint from itself, a contradiction. In the next section we reprove this fact in a more general context.

We now check the last clause of the definition of turbulence.

Let $U$ be a neighborhood of the identity in $G$, and $V_{0}$ be a neighborhood of an ergodic transformation $T$. Let $V \subset V_{0}$ be a neighborhood of $T$ of the form $N\left(A_{0}, \ldots, A_{l}, \rho\right)$. Let $S \in V$ be ergodic and $W$ be a neighborhood of $S$.

By Lemma 9 , we can replace $S$ by an $S^{\prime} \in W$ that has the same orbits almost everywhere that $T$ does and then replace $W$ by a basic open neighborhood $W^{\prime} \subset W$ of $S^{\prime}$. Hence without loss of generality we can assume that $S$ has the same orbits almost everywhere as $T$ does.

Apply the claim. By choosing $\epsilon$ small enough, (1) ensures that each $g_{i} \in U$. If we choose $\delta$ small enough, (2) guarantees that each $T_{i} \in V$ and (3) guarantees that $T_{n} \in W$.

We now prove the claim. Choose now an $M$ so large that the collection $L_{0}$ of $x$ for which $\left\{S x, S^{-1} x\right\}$ is not a subset of the partial orbit $\left\{T^{-M+1} x, T^{-M+2} x, \ldots, T^{-1} x\right.$, $\left.x, T x, \ldots, T^{M-1} x\right\}$ has measure less than $\delta / 3$.

Let $N$ be so large that $2 M / N<\delta / 3$, and choose an $(N, \delta / 3)$ Rokhlin tower with base $B$. Let $L_{1}$ be the union of the first and last $M$ levels of the tower. Then $L_{1}$ has measure at most $\delta / 3$. Let $L_{2}$ be the collection of $x$ not in the levels of the Rokhlin tower. Then by assumption the measure of $L_{2}$ is less than $\delta / 3$ and hence the measure of $L_{0} U$ $L_{1} \cup L_{2}$ is less than $\delta$.

Let $y \in B$. Then for all $x \in\left\{T^{i} y: M \leq i \leq N-M\right\} \backslash L_{0}$, both $S x$ and $S^{-1} x$ belong to $\left\{T^{i} y: 0 \leq i \leq N-1\right\}$. 
We can choose a cyclic permutation $\sigma_{y}$ of $\{0, \ldots, N-1\}$ such that for all $i, j<$ $N-1$, if $S\left(T^{i} y\right)=T^{j} y$, then $\sigma_{y}(i)=j$. For each permutation $\sigma$ of $\{0, \ldots, N-1\}$, the collection $B_{\sigma}=\left\{y: \sigma_{y}=\sigma\right\}$ is measurable and the sets $B_{\sigma}$ partition $B$.

Partition each $B_{\sigma}$ into sets $B_{\sigma, k}, k<k_{\sigma}$, such that the measure of each $B_{\sigma, k}$ is less than $\epsilon / N$. Let $\tau_{\sigma}$ be a permutation of $\{0, \ldots, N-1\}$ such that $\tau_{\sigma} \sigma \tau_{\sigma}^{-1}$ is the cyclic permutation $(0,1, \ldots, N-1)$ and define $u_{\sigma, k}$ on $\bigcup_{i<N} T^{i} B_{\sigma, k}$ by setting

$$
u_{\sigma, k}\left(T^{i} y\right)=T^{\tau_{\sigma}^{(i)}} y
$$

for $y \in B_{\sigma, k}$ and $u_{\sigma, k}(x)=x$ for $x \notin \bigcup_{i<N} T^{i} B_{\sigma, k}$.

Let $g_{1}, \ldots, g_{n-1}$ enumerate the $u_{\sigma, k}$ 's (in any order). Note that it is clear that condition (1) is satisfied. Moreover, since the collections of $x$ moved by the various $u_{\sigma, k}$ 's are pairwise disjoint, the $u_{\sigma, k}$ 's commute.

By the choice of $\tau_{\sigma}$, for all $x \in \bigcup_{M \leq i \leq N-M} T^{i} x \backslash L_{0}$,

$$
u_{\sigma, k}(x) T(x) u_{\sigma, k}^{-1}(x)=S(x),
$$

hence for all $x \notin L_{0} \cup L_{1} \cup L_{2}, T_{i}(x) \in\{S(x), T(x)\}$ and hence (2) is satisfied.

Moreover, for all $x \notin L_{0} \cup L_{1} \cup L_{2}$ that are moved by any $g_{i}$, we have $T_{i+1}(x)=S(x)$. In particular $T_{n}(x)=S(x)$ except on a set of measure less than $\delta$.

We remark that a fact analogous to Claim 14 is true for finitely generated amenable groups.

\section{General discrete groups}

In this section we discuss this theorem in the context of countable discrete group actions by measure preserving transformations. We will prove the result analogous to Theorem 12 for the case of monotilable amenable groups and discuss the situation for general groups.

We begin by fixing a countable group $H$. We put a topology on the space of $H$ actions on $[0,1]$ by measure preserving transformations. (As usual we will identify actions that agree on a set of full measure.) For each $H$-action $\mathcal{A}$ on $[0,1]$ and $g \in H$ we will denote the measure preserving transformation determined by the action and $g$ as $g^{\mathcal{A}}$. Fix such an action $\mathcal{A}$. We describe a basic open neighborhood of $\mathcal{A}$. For each $\epsilon>0, g_{1}, \ldots, g_{n} \in H$ and measurable partition $\left\{A_{1}, \ldots, A_{k}\right\}$ we take as a basic open neighborhood $N\left(\mathcal{A}, g_{1}, \ldots, g_{n}, A_{1}, \ldots, A_{k}, \epsilon\right)$ the set

$$
\left\{\mathcal{B}: \mu\left(g_{i}^{\mathcal{A}} A_{j} \triangle g_{i}^{\mathcal{B}} A_{j}\right)<\epsilon \text { for all } i, j\right\} .
$$

(We note that if $H$ is finitely generated with generators $\left\{h_{1}, \ldots, h_{n}\right\}$, then the collection of neighborhoods determined by taking the $g_{i}$ 's to be the $h_{i}$ 's forms a base for the topology.)

As before this is a Polish topology. The collection of free ergodic actions of a countable amenable group $H$ form a dense $\mathcal{G}_{\delta}$ set in the collection of all actions and hence a Polish space (see [3]).

If $T$ is a measure preserving transformation and $\mathcal{A}$ is an $H$-action by measure preserving transformation on $[0,1]$ then we let $\mathcal{A}^{\prime}=T \mathcal{A}$ be the $H$-action defined by setting 
$g^{\mathcal{A}^{\prime}}=T g^{\mathcal{A}} T^{-1}$. (We will sometimes write this as $\mathcal{A}^{\prime}=T \mathcal{A} T^{-1}$.) This defines an action of the group of measure preserving transformations on the space of $H$-actions and the orbits coincide with isomorphism classes of $H$-actions.

Theorem 15. Suppose that $H$ is an amenable group. Then the action of the measure preserving transformations on the ergodic $H$-actions is turbulent.

A closely related theorem is:

Theorem 16. Suppose that $H$ is an amenable group and $\mathcal{A}$ is an ergodic action of $H$. Then the collection of actions $\mathcal{B}$ that have the same orbits almost everywhere as $\mathcal{A}$ does is dense in the space of $H$-actions.

We now give the proof of this theorem in the case that the amenable group is monotilable. The general result follows tediously using the quasi-tiling techniques of [13]. We begin by discussing monotilable groups. For an exposition about monotilable groups we refer the reader to [14].

Definition 17. An amenable group $H$ is monotilable iff there is a Følner sequence $\left\langle F_{n}\right.$ : $n \in \mathbb{N}\rangle$ and a collection $\left\{c_{n}^{i}: i, n \in \mathbb{N}\right\}$ such that for each fixed $n,\left\{F_{n} c_{n}^{i}: i \in \mathbb{N}\right\}$ is a partition of $H$. The sequence $\left\langle F_{n}: n \in \mathbb{N}\right\rangle$ will be called $a$ tiling sequence. A set $B$ such that there are $\left\{c^{i}: i \in \mathbb{N}\right\}$ such that $\left\{B c^{i}: i \in \mathbb{N}\right\}$ is a partition of $H$ is called a tiling set and the $\left\{c^{i}: i \in \mathbb{N}\right\}$ are the centers of the tiling.

All elementary groups are monotilable and it is an open question whether every amenable group is monotilable. For our purposes the main advantage of monotilability is an easily usable version of Rokhlin's Lemma.

If $\Gamma, F \subset H$, we will call the set $\{h \in F: g h \notin F$ for some $g \in \Gamma\}$ the inner $\Gamma$ boundary of $F$. The $\Gamma$-interior of $F$ is the collection of elements of $F$ not in the inner boundary.

Rokhlin's Lemma III ([13]). Suppose that $H$ is a monotilable group acting freely and ergodicly on $[0,1]$ by measure preserving transformations with tiling sequence $\left\langle F_{n}\right.$ : $n \in \mathbb{N}\rangle$. Then for all $N$ large enough $\epsilon>0$ and any measurable subsets $\left\{A_{1}, \ldots, A_{n}\right\}$ of $[0,1]$ there is a set $B \subset[0,1]$ such that:

(1) The sets $\left\{g B: g \in F_{N}\right\}$ are pairwise disjoint.

(2) The measure of $\bigcup_{g \in F_{N}} g B$ is at least $1-\epsilon$.

(3) For all $y \in B$ and $j<n$,

$$
\left|\#\left\{g \in F_{N}: g(y) \in A_{j}\right\} /\right| F_{N}\left|-\mu\left(A_{j}\right)\right|<\epsilon .
$$

We will call this an $\left(F_{N}, \epsilon\right)$ Rokhlin tower for $\left\{A_{1}, \ldots, A_{n}\right\}$. We will refer to $B$ as the base and $X \backslash \bigcup_{g \in F_{N}} g B$ as the remainder of the tower.

Given an $(F, \epsilon)$ Rokhlin tower with base $B$, we will call the set $\bigcup\{h B: h$ is in the inner $\Gamma$-boundary of $F\}$ the $\Gamma$-boundary of the tower, and the set $\bigcup\{h B: h$ is in the $\Gamma$-interior of $F\}$ the $\Gamma$-interior of the tower. Clearly if the boundary is a small fraction of $F$ and $\epsilon$ is small, the vast majority of the unit interval is in the interior of the tower. 
Fix an $(F, \epsilon)$ Rokhlin tower with base $B$. Suppose that $\mathbb{P}=\left\{P_{\tau}: \tau \in S\right\}$ is a partition of the base of $B$ where $S$ is a collection of permutations of a subset of $F$. For $y \in P_{\tau}$ and $h$ in the domain of $\tau$, let $T_{\mathbb{P}}(h y)=\tau(h) y$, and let $T_{\mathbb{P}}$ be the identity on the remainder of the tower. Then $T_{\mathbb{P}}$ is a measure preserving transformation that locally permutes the levels of the tower and preserves the $F$-orbits inside the tower. We will call $T_{\mathbb{P}}$ the transformation induced by the partition and $S$. We will let $T_{\tau}$ denote the restriction of $T$ to the collection of $F$-orbits of $P_{\tau}$.

Remark 18. For any $\mathcal{A}$ the $H$-action $\mathcal{A}^{\prime}=T_{\mathbb{P}} \mathcal{A} T_{\mathbb{P}}{ }^{-1}$ has the same orbits almost everywhere as $\mathcal{A}$ does.

We now prove Theorems 15 and 16 simultaneously, combining in one proof the two explicit steps from the case of $\mathbb{Z}$ :

Proof of Theorems 15 and 16. Let $H$ be an amenable group and let $X$ be the space of free ergodic $H$-actions by measure preserving transformations on $[0,1]$. As remarked above, Glasner and King showed in [3] that the free ergodic actions of $H$ are a dense $\mathcal{G}_{\delta}$ set in the space of all actions of $H$. In particular, it is a Polish space with the induced topology. We let the group of measure preserving transformations act on $[0,1]$ by conjugation.

Claim 19. Every orbit of a free action of $H$ is dense in $X$.

Proof. This follows immediately from Rokhlin's Lemma: If $\mathcal{A}$ and $\mathcal{B}$ are $H$-actions, fix a neighborhood of $\mathcal{B}, N\left(\mathcal{B}, g_{1}, \ldots, g_{n}, A_{1}, \ldots, A_{k}, \epsilon\right)$. Let $F_{N}$ be an element of the tiling sequence such that for all $i,\left|g_{i} F_{N} \triangle F_{N}\right| /\left|F_{N}\right|<\epsilon / 2$. Apply Rokhlin's Lemma for $F_{N}, \epsilon / 2$ and the actions $\mathcal{A}, \mathcal{B}$ to find sets $B_{\mathcal{A}}, B_{\mathcal{B}}$. (For these purposes the last clause of Rokhlin's Lemma is irrelevant.) We can assume that $B_{\mathcal{A}}$ and $B_{\mathcal{B}}$ have the same measure. Let $T_{0}: B_{\mathcal{A}} \rightarrow B_{\mathcal{B}}$ be measure preserving and extend $T_{0}$ to $\bigcup_{g \in F_{N}} g^{\mathcal{A}} B_{\mathcal{A}}$ by setting $T_{0}\left(g^{\mathcal{A}} y\right)=g^{\mathcal{B}} T_{0}(y)$ for $y \in B_{\mathcal{A}}$ and $g \in F_{N}$. Finally, extend $T_{0}$ to a measure preserving transformation $T$ of $[0,1]$ on the remainder of the Rokhlin tower arbitrarily. Then for each $i$, the measure of the set $\left\{x: \operatorname{Tg}_{i}^{\mathcal{A}} T^{-1}(x) \neq g^{\mathcal{B}}(x)\right\}$ is less than $\epsilon$. In particular, for all $i, j,\left(T g_{i}^{\mathcal{A}} T^{-1}\right)\left(A_{j}\right) \triangle g_{i}^{\mathcal{B}}\left(A_{j}\right)$ has measure less than $\epsilon$.

Claim 20. The orbit of every $H$-action is meager.

Proof. (We note that this claim does not use monotilability for its proof.) Since every orbit of $H$ is invariant under the group of measure preserving transformations, and dense, standard 0-1 laws show that a non-meager orbit must be comeager. (See e.g. [3] or [6] for a proof of this classical result.)

Fix an action $\mathcal{A}$ of the group $H$. We recall the definition of entropy of the action. If $\left\langle F_{n}: n \in \omega\right\rangle$ is a Følner sequence for $H$, and $\mathbb{P}$ is a partition of $[0,1]$, we let $\bigvee_{F_{n}} \mathbb{P}$ be the partition generated by $\left\{h \mathbb{P}: h \in F_{n}\right\}$ and define

$$
h_{n}(\mathcal{A}, \mathbb{P})=-\sum\left\{\mu(a) \log (\mu(a)): a \in \bigvee_{F_{n}} \mathbb{P}\right\} /\left|F_{n}\right|
$$

Note that $h_{n}$ is a continuous function in $\mathcal{A}$. 
The entropy of $\mathcal{A}$ with respect to $\mathbb{P}$ is defined by $h(\mathcal{A}, \mathbb{P})=\lim _{n \rightarrow \infty} h_{n}(\mathcal{A}, \mathbb{P})$ and the entropy of $\mathcal{A}$ is given by $h(\mathcal{A})=\sup \{h(\mathcal{A}, \mathbb{P}): \mathbb{P}$ is a finite partition $\}$.

Subclaim. For all $\gamma \geq 0,\{\mathcal{A}: h(\mathcal{A})<\gamma\}$ is a dense $\mathcal{G}_{\delta}$ set. In particular, the set of zero entropy actions of an amenable group is a dense $\mathcal{G}_{\delta}$ set.

Proof. (This proof is due to Rudolph.) It can be shown that $h(\mathcal{A}, \mathbb{P})$ is a continuous function of $\mathbb{P}$ in the sense that for all $k \in \mathbb{N}$ and $\epsilon>0$ there is a $\delta>0$ such that if $\mathbb{P}=\left\{P_{1}, \ldots, P_{k}\right\}$ and $\mathbb{Q}=\left\{Q_{1}, \ldots, Q_{k}\right\}$ are two partitions with $\sum \mu\left(P_{i} \triangle Q_{i}\right)<\delta$ then $|h(\mathcal{A}, \mathbb{P})-h(\mathcal{A}, \mathbb{Q})|<\epsilon$. Moreover, entropy is monotone in the sense that if $\mathbb{P}$ refines $\mathbb{Q}$ then $h(\mathcal{A}, \mathbb{Q}) \leq h(\mathcal{A}, \mathbb{P})$. From this we can deduce that

$$
h(\mathcal{A})=\sup \{h(\mathcal{A}, \mathbb{P}): \mathbb{P} \text { is a finite partition with rational endpoints }\} .
$$

From this it is routine to see that for all $\gamma,\{\mathcal{A}: h(\mathcal{A})<\gamma\}$ is a $\mathcal{G}_{\delta}$ set.

For each positive $\gamma$, choose a sequence $\left\langle a_{n}: n \in \mathbb{N}\right\rangle$ of positive numbers such that $\sum a_{n}=1$ and $-\sum_{n \in \mathbb{N}} a_{n} \log \left(a_{n}\right)<\gamma$. This makes $\mathbb{N}$ into an atomic measure space and if we endow $X=\mathbb{N}^{H}$ with the product measure we get a Lebesgue space. Let $H$ act on $\mathbb{N}^{H}$ by $h f(h)=f\left(h^{-1} g\right)$. This action is ergodic and has entropy less than $\gamma$.

We will call the action of $H$ on $\mathbb{N}^{H}$ with the measure given above the Bernoulli action with coefficients $\left\{a_{n}\right\}$.

Del Junco proves the following result for $\mathbb{Z}$-actions, but the proof carries over directly to amenable actions:

Theorem 21 (del Junco [9]). Suppose that $\mathcal{A}$ is an $H$-action. Then $\{\mathcal{B}: \mathcal{B}$ is disjoint from $\mathcal{A}$ \} is a $\mathcal{G}_{\delta}$ set.

Moreover, classical methods show that if $\mathcal{A}$ has zero entropy then $\mathcal{A}$ is disjoint from any Bernoulli action.

We now finish the proof of Claim 20 If $\mathcal{A}$ were an action with non-meager orbit then the orbit of $\mathcal{A}$ would have to be comeager. In particular $\mathcal{A}$ would have to have zero entropy. On the other hand, the transformations disjoint from $\mathcal{A}$ are a non-empty $\mathcal{G}_{\delta}$ set (because they contain Bernoulli actions). Since any non-empty, conjugation invariant $\mathcal{G}_{\delta}$ set must be dense we see that $\mathcal{A}$ must be disjoint from itself. This contradiction proves Claim 20.

We will prove the following:

Subclaim. Suppose that $\epsilon>0$ and let $W=N\left(\mathcal{B}, g_{0}, \ldots, g_{n-1}, B_{0}, \ldots, B_{k-1}, \eta\right)$ be a basic open neighborhood. Then there is a $p$ and a sequence of measure preserving transformations $u_{i}, i<p$, such that:

(1) The set $\left\{x: u_{i}(x) \neq x\right\}$ has measure less than $\epsilon$.

(2) If $\mathcal{A}_{0}=\mathcal{A}$ and $\mathcal{A}_{i+1}=u_{i} \mathcal{A}_{i}$ then $g_{j}^{\mathcal{A}_{i}}(x) \in\left\{g_{j}^{\mathcal{A}}(x), g_{j}^{\mathcal{A}_{p}}(x)\right\}$ for all $i, j, x$.

(3) $\mathcal{A}_{p}(x) \in W$.

(4) Each $\mathcal{A}_{i}$ has the same orbits almost everywhere as $\mathcal{A}$ does. 
To see the subclaim suffices for Theorem 16 take any ergodic $\mathcal{A}, \mathcal{B}$, and a neighborhood $W$ of $\mathcal{B}$. Applying the subclaim, we see that $\mathcal{A}_{p}$ has the same orbits almost everywhere as $\mathcal{A}$ does and is in the neighborhood of $\mathcal{B}$.

For the proof of Theorem 15 we use the subclaim to check the last clause of the hypothesis of Lemma 4 Fix an action $\mathcal{A}$, a neighborhood $V=N\left(\mathcal{A}, g_{0}, \ldots, g_{m}, A_{0}, \ldots\right.$, $\left.A_{k-1}, \rho\right)$, an ergodic action $\mathcal{B} \in V$, a neighborhood $W=N\left(\mathcal{B}, h_{0}, \ldots, h_{n}, B_{0}, \ldots\right.$, $\left.B_{l-1}, \eta\right)$ and a neighborhood $U$ of the identity in the group of measure preserving transformations. We note that without loss of generality we can assume that $m=n$, and $h_{j}=g_{j}$ for $j<n$. Moreover, the partition $\left\{B_{0}, \ldots, B_{l-1}\right\}$ refines $\left\{A_{0}, \ldots, A_{k-1}\right\}$ and $\eta<\rho$.

Applying the subclaim with $\epsilon$ small enough we get a sequence of $u_{i}$ 's such that each $u_{i}$ belongs to $U$. If we take $\delta$ small enough and use the fact that $\mathcal{A}_{p}$ belongs to $V$, clause (2) of the subclaim shows that each $\mathcal{A}_{i}$ belongs to $V$.

To prove the subclaim, we begin by constructing an action $\mathcal{A}^{\prime}$ which will eventually become $\mathcal{A}_{p}$. Having done this the proof follows the proof of Claim 14. The "prescription" for finding $\mathcal{A}^{\prime}$ goes as follows: we take a very large element $F$ of a tiling Følner sequence, and two Rokhlin towers for $F$, one for $\mathcal{A}$ and one for $\mathcal{B}$. We "purify" by breaking the bases of the towers into sets such that on each set $P$ and for each $h \in F, h P$ is either disjoint from or contained in the partition determined by the $B_{i}$ 's and the $g_{j}^{\mathcal{B}} B_{i}$ 's. We then permute $F P$ by some $\tau$ so that $\mathcal{A}^{\prime}=T_{\tau} \mathcal{A} T_{\tau}^{-1}$ behaves exactly the same way as $\mathcal{B}$ does with respect to the $B_{i}$ 's and their images under $g_{j}^{\mathcal{B}}$. Since the vast majority of mass lies in the interior of $F P, \mathcal{A}^{\prime}$ is in the neighborhood $W$.

We now follow the prescription more formally. Let $F_{M}$ be an element of the tiling sequence for $H$ such that for all $j,\left|g_{j} F_{M} \triangle F_{M}\right| /\left|F_{M}\right|<\eta / 6$, i.e. the boundary of $F_{M}$ has size less than $(\eta / 6)\left|F_{M}\right|$.

Let $\mathcal{Q}$ be the partition of $[0,1]$ generated by $\left\{B_{0}, \ldots, B_{l-1}\right\}$ and $\left\{g_{j}^{\mathcal{B}} B_{i}: i<l\right.$ and $j<n$ \}. Let $B^{\mathcal{A}}$ and $B^{\mathcal{B}}$ be bases of $\left(F_{M}, \eta / 6\right)$ Rokhlin towers for the partition $\mathcal{Q}$, and the systems $\mathcal{A}$ and $\mathcal{B}$ respectively. We can assume that the measures of $B^{\mathcal{A}}$ and of $B^{\mathcal{B}}$ are equal. We let $L_{0}$ be the remainder $[0,1] \backslash \bigcup_{h \in F_{M}} h^{\mathcal{A}} B^{\mathcal{A}}$. Let $L_{1}$ be the boundary of the tower. Then the measures of both $L_{0}$ and $L_{1}$ are less than or equal to $\eta / 6$ and $[0,1] \backslash L_{0} \cup L_{1}$ is the interior of the tower.

For each atom $a$ of $\mathcal{Q}$ and $y \in B^{\mathcal{A}},\left|\#\left\{h \in F_{M}: h^{\mathcal{A}}(y) \in a\right\} /\right| F_{M}|-\mu(a)|<$ $\eta / 6$ and similarly for all $y \in B^{\mathcal{B}}$. By shrinking the bases $B^{\mathcal{A}}$ and $B^{\mathcal{B}}$, an arbitrarily small amount, we can preserve the fact that we have $\left(F_{M}, \eta / 6\right)$ Rokhlin towers and find partitions $P_{0}^{\mathcal{A}}, \ldots, P_{r-1}^{\mathcal{A}}$ and $P_{0}^{\mathcal{B}}, \ldots, P_{r-1}^{\mathcal{B}}$ of the bases $B^{\mathcal{A}}$ and $B^{\mathcal{B}}$ such that:

(1) For all $s<r$ the measure of $P_{s}^{\mathcal{A}}$ is equal to the measure of $P_{s}^{\mathcal{B}}$.

(2) For all $s<r$ and $h \in F_{M}$ there are atoms $a, b$ of $\mathcal{Q}$ such that $h^{\mathcal{A}} P_{s}^{\mathcal{A}} \subset a$ and $h^{\mathcal{B}} P_{s}^{\mathcal{B}} \subset b$.

(3) For each $s$, there is a permutation $\sigma=\sigma(s)$ of $F_{M}$ such that for all but $(2 \eta / 6)\left|F_{M}\right|$ many $h \in F_{M}$ and all atoms $a$ of $\mathcal{Q}$,

$$
h^{\mathcal{A}} P_{s}^{\mathcal{A}} \subset a \text { iff } \quad \sigma(h)^{\mathcal{B}} P_{s}^{\mathcal{B}} \subset a
$$


Let $S=\{\sigma(s): s<r\}$. Let $T$ be the measure preserving transformation induced by the partition $\left\{P_{0}^{\mathcal{A}}, \ldots, P_{r-1}^{\mathcal{A}}\right\}$ and $S=\{\sigma(s): s<r\}$ (as in the remarks before Remark 18. Define $\mathcal{A}^{\prime}$ to be $T \mathcal{A} T^{-1}$. Then by Remark $18, \mathcal{A}^{\prime}$ has the same orbits almost everywhere as $\mathcal{A}$ does.

We now argue that $\mathcal{A}^{\prime}$ is in the neighborhood $W$. For each $s$, we consider the levels of the Rokhlin tower above $P_{s}^{\mathcal{A}}$. We will call a level $h^{\mathcal{A}} P_{s}^{\mathcal{A}}$ bad if for the atom $a \in \mathcal{Q}$ with $h^{\mathcal{A}} P_{s}^{\mathcal{A}} \subset a$ we have $\sigma_{s}(h)^{\mathcal{B}} P_{s}^{\mathcal{B}} \not \subset a$.

For each $x$ in the interior of the tower above $P_{s}^{\mathcal{A}}$, if $x \in g_{j}^{\mathcal{A}^{\prime}} B_{i} \triangle g_{j}^{\mathcal{B}} B_{i}$ then either $x$ or $\left(g_{j}^{\mathcal{A}^{\prime}}\right)^{-1} x$ belongs to a bad level of the tower over $P_{s}^{\mathcal{A}}$, or else $\left(g_{j}^{\mathcal{A}^{\prime}}\right)^{-1} x$ belongs to $L_{0}$. Hence the measure of the intersection of the tower over $P_{s}^{\mathcal{A}}$ with $g_{j}^{\mathcal{A}^{\prime}} B_{i} \triangle g_{j}^{\mathcal{B}} B_{i}$ is at most twice the measure of the union of the bad levels of the tower above $P_{s}^{\mathcal{A}}$ plus the measure of $\left\{x \in L_{0}: g_{j}^{\mathcal{A}^{\prime}}(x) \in P_{s}^{\mathcal{A}}\right\}$.

Let $L_{3}^{s}$ be the union of the bad levels of the tower over $P_{s}^{\mathcal{A}}$. Then the measure of $L_{3}^{s}$ is less than $2(\eta / 6)\left|F_{M}\right| \mu\left(P_{s}^{\mathcal{A}}\right)$. Since $\bigcup_{s} P_{s}^{\mathcal{A}}=B^{\mathcal{A}}, \bigcup_{s} P_{s}^{\mathcal{A}}$ has measure less than $1 /\left|F_{M}\right|$. Thus if $L_{3}=\bigcup_{s<r} L_{3}^{s}$ then the measure of $L_{3}$ is less than $2(\eta / 6)$.

Thus for each $j$ and $i$, the intersection of $g_{j}^{\mathcal{A}^{\prime}} B_{i} \triangle g_{j}^{\mathcal{B}} B_{i}$ with the interior of the tower $\bigcup_{h \in F_{M}} h B^{\mathcal{A}}$ has measure at most $2 \mu\left(L_{3}\right)+\mu\left(L_{0}\right)<5(\eta / 6)$. Hence the total measure of $g_{j}^{\mathcal{A}^{\prime}} B_{i} \triangle g_{j}^{\mathcal{B}} B_{i}$ is less than $\mu\left(L_{0}\right)+\mu\left(L_{1}\right)+2 \mu\left(L_{3}\right)=\eta$.

Divide each $P_{s}$ into finite partitions $\left\{P_{s, t}\right\}_{t}$ such that the measure of each $P_{s, t}$ is less than $\epsilon /\left|F_{M}\right|$. Define $u_{s, t}$ by setting it equal to $T$ on the orbits of $P_{s, t}$ and the identity otherwise. Then:

- The measure of $\left\{x: u_{s, t}(x) \neq x\right\}$ is less than $\epsilon$.

- The $u_{s, t}$ 's commute.

- $T$ is equal to the product of all of the $u_{s, t}$.

In particular, if we enumerate the $u_{s, t}$ 's as $\left\langle u_{0}, u_{1}, \ldots, u_{p-1}\right\rangle$, then $\mathcal{A}_{p}=\mathcal{A}^{\prime}$ and we have proven the subclaim.

\subsection{General amenable groups}

If the amenable group $H$ is not known to be monotilable the proof is carried out by using the quasi-tiling machinery of [13]. Roughly speaking, for any free action of an amenable group $H$ on $[0,1]$ by measure preserving transformations, this machinery provides a finite collection of disjoint Rokhlin towers parametrized by different Følner sets that fill up most of the space. The proof that we have given can then be carried out, mutatis mutandis, with these towers replacing the single tower of the monotilable case. We shall content ourselves with formulating the generalization of the Rokhlin Lemma for the general case and leave the details of the proof to the reader.

We will first formulate the basic Rokhlin lemma and then the version that is needed which incorporates an application of the ergodic theorem. 
If we are given finite subsets $K, F$ of $H$ and a $\delta>0$, then we will say that $F$ is $(K, \delta)$-invariant if $\left|\bigcup_{k \in K} k F \triangle F\right|<\delta|F|$. For $K$ with $K=K^{-1}$ and $e \in K$, this definition is equivalent to the one given in [13].

Rokhlin's Lemma IV ([13]). Suppose that $H$ is an amenable group, and a finite subset $K$ of $H$ and $\delta, \epsilon>0$ are given. Then there are sets $\left\{F_{1}, \ldots, F_{k}\right\}$ that are $(K, \delta)$-invariant and numbers $b_{1}, \ldots, b_{k} \in[0,1]$ with $\sum b_{i}>1-\epsilon$ so that for any free ergodic action of $H$ on $[0,1]$ by measure preserving transformations there are sets $\left\{B_{1}, \ldots, B_{k}\right\}$ in the unit interval that satisfy:

(1) For each $i$ the sets $\left\{f B_{i}: f \in F_{i}\right\}$ are disjoint.

(2) The sets $\left\{F_{i} B_{i}: 1 \leq i \leq k\right\}$ are pairwise disjoint.

(3) The measure of $F_{i} B_{i}$ is $b_{i}$.

It is important to note that the sets $F_{i}$ depend only on $K, \delta$ and $\epsilon$ and not on the specific action. Also the measure of each of the $F_{i} B_{i}$ depends only on $i$ and not on the action. For these addenda see the proof of Theorem 5 in II. $\$ 2$ of [13] and Remark 3 in II. $\$ 3$ there.

For the final version of the lemma assume that in addition to the action of $H$ we are also given a finite number of measurable subsets $A_{j}$ in $[0,1]$. Then we have the following:

Rokhlin's Lemma V ([13]). Suppose that $H$ is an amenable group, and a finite subset $K$ of $H$ and $\delta, \epsilon>0$ are given. Then there are sets $\left\{F_{1}, \ldots, F_{k}\right\}$ that are $(K, \delta)$-invariant and numbers $b_{1}, \ldots, b_{k} \in[0,1]$ with $\sum b_{i}>1-\epsilon$ so that for any free ergodic action of $H$ on $[0,1]$ by measure preserving transformations there are sets $\left\{B_{1}, \ldots, B_{k}\right\}$ in the unit interval that satisfy:

(1) For each $i$ the sets $\left\{f B_{i}: f \in F_{i}\right\}$ are disjoint.

(2) The sets $\left\{F_{i} B_{i}: 1 \leq i \leq k\right\}$ are pairwise disjoint.

(3) The measure of $F_{i} \bar{B}_{i}$ is $b_{i}$.

(4) For all $y \in \bigcup B_{i}$ and $j<n$,

$$
\left|\#\left\{g \in F_{N}: g(y) \in A_{j}\right\} /\right| F_{N}\left|-\mu\left(A_{j}\right)\right|<\epsilon .
$$

As before we note that the sets $F_{i}$ depend only on $K, \delta$ and $\epsilon$ and not on the specific action as do the measures of the $F_{i} B_{i}$.

\section{References}

[1] Feldman, J.: Borel structures and invariants for measurable transformations. Proc. Amer. Math. Soc. 46, 383-394 (1974) Zbl 0292.28008 MR 50 \#7479

[2] Foreman, M.: A descriptive view of ergodic theory. In: Descriptive Set Theory and Dynamical Systems (Marseille-Luminy, 1996), London Math. Soc. Lecture Note Ser. 277, Cambridge Univ. Press, Cambridge, 87-171 (2000) Zbl 0962.03043 MR 2001h:37003

[3] Glasner, E., King, J.: A zero-one law for dynamical properties. In: Topological Dynamics and Applications (Minneapolis, MN, 1995), Contemp. Math. 215, Amer. Math. Soc., Providence, RI, 231-242 (1998) Zbl 0909.28014 MR 99d:28039

[4] Halmos, P. R.: Lectures on Ergodic Theory. Chelsea, New York (1960) Zbl 0096.09004 MR 22 \#2677 
[5] Harrington, L. A., Kechris, A. S., Louveau, A.: A Glimm-Effros dichotomy for Borel equivalence relations. J. Amer. Math. Soc. 3, 903-928 (1990) Zbl 0778.28011 MR 91h:28023

[6] Hjorth, G.: Classification and Orbit Equivalence Relations. Math. Surveys Monographs 75, Amer. Math. Soc., Providence, RI (2000) Zbl 0942.03056 MR 2000k:03097

[7] Hjorth, G.: Non-smooth infinite dimensional group representations. Notes at: http://www.math.ucla.edu/greg/

[8] Hjorth, G.: On invariants for measure preserving transformations. Fund. Math. 169, 51-84 (2001) Zbl 0990.03036 MR 2002j:03048

[9] del Junco, A.: Disjointness of measure-preserving transformations, minimal self-joinings and category. In: Ergodic Theory and Dynamical Systems, I (College Park, MD, 1979-80), Progr. Math. 10, Birkhäuser Boston, 81-89 (1981) Zbl 0467.28011 MR 82m:28035

[10] von Neumann, J.: Zur Operatorenmethode in der klassischen Mechanik. Ann. of Math. (2) 33, 587-642 (1932) Zbl 0005.12203 MR 1503078

[11] Ornstein, D. S.: Some new results in the Kolmogorov-Sinai theory of entropy and ergodic theory. Bull. Amer. Math. Soc. 77, 878-890 (1971) Zbl 0990.03036 MR 44 \#5431

[12] Ornstein, D. S., Weiss, B.: Geodesic flows are Bernoullian. Israel J. Math. 14, 184-198 (1973) Zbl 0256.58006 MR 48 \#4272

[13] Ornstein, D. S., Weiss, B.: Entropy and isomorphism theorems for actions of amenable groups. J. Anal. Math. 48, 1-141 (1987) Zbl 0637.28015 MR 88j:28014

[14] Weiss, B.: Monotileable amenable groups. In: Topology, Ergodic Theory, Real Algebraic Geometry, Amer. Math. Soc. Transl. 202, Amer. Math. Soc., 257-262 (2001) Zbl 0982.22004 MR 2001m:22014 